\title{
Spatial memory in rats: Resistance to retroactive interference
}

\author{
WILLIAM S. MAKI, SUSAN BROKOFSKY, and BRUCE BERG \\ North Dakota State University, Fargo, North Dakota 58105
}

\begin{abstract}
Treatments that interfere with animals' short-term retention (e.g., in delayed matching-tosample) were studied using a spatial memory task. Rats performed in an eight-arm radial maze in which choosing each arm without repetition was the optimal behavior. Performances were interrupted between fourth and fifth choices for a delay of $15 \mathrm{sec}$ to $2 \mathrm{~min}$. A variety of events occurring during the delay interval did not disrupt memories for prior choices (as assessed by the accuracy of postdelay choices). The ineffective treatments included variations in visual and auditory environments, removal from the maze, food consumed during the delay, a distinctive odor added to the maze, or combinations of these manipulations. Additionally, performance on another spatial task (a four-arm maze) during the delay between Choices 4 and 5 did not interfere with performance in the eight-arm maze. These findings suggest that rats' memories for spatial locations are immune to retroactive interference, at least within the range of conditions reported, and that the rat can successfully segregate memories for spatial locations established in different contexts.
\end{abstract}

Olton $(1977,1978)$ reported that rats display a remarkable memory for spatial locations in a radialarm maze. In that situation, each of several arms is baited with food and the rat is allowed to choose among the arms, always returning to the center of the maze between choices. Optimal behavior consists of choosing each arm once without repetitions, because once food is depleted at one location it is not replenished. In eight-arm versions of the radial-arm maze, accuracies have been reported in excess of $95 \%$ correct choices. A finding of particular interest is that these high levels of accuracy are maintained even if the rat is prevented from responding, by confinement to the center of the maze, for 2 min after the first four choices (Olton \& Samuelson, 1976). Moreover, removing the rat from the maze for $1 \mathrm{~min}$ after three choices does not impair accuracy of subsequent choices (Zoladek \& Roberts, 1978). The latter finding is particularly impressive; most certainly removing a rat from a maze subjects it to an aggregation of stimuli (visual, auditory, tactile, vestibular, and olfactory) which, nevertheless, seems not to interfere with the retention of prior choices. These findings invite the conjecture that rats' spatial memories are quite durable, perhaps because they are resistant to retroactive interference (RI) from incidental environmental events. In contrast, in delayed matching-tosample (DMTS) tests of memory, monkeys, dolphins, and pigeons all show detrimental effects of incidental stimulation during retention intervals (D'Amato, 1973; Herman, 1975; Maki, Moe, \& Bierley, 1977). The present experiments were designed to evaluate the effects of delay-interval stimulation on rats' memories for spatial locations in the radial-arm maze. The aim was to determine the extent to which such memories are susceptible to RI.

\section{EXPERIMENT 1}

\section{General Method}

Subjects. Male Holtzman albino rats $(N=12)$ were about 120 days old at the start of the experiment. The rats were housed individually in a room illuminated from $0800-2000 \mathrm{~h}$, and provided free access to water. Their diets consisted of $45-\mathrm{mg}$ Noyes pellets (obtained as rewards during training sessions); Purina Lab Chow was provided immediately after training sessions in quantities sufficient to maintain them at $85 \%$ of their preexperimental body weights. In Experiment le, the rats also consumed small portions of chocolate Oreo cookies. Throughout, the rats were allowed to gain an additional $5 \mathrm{~g} /$ week.

Apparatus. The eight-arm maze was modeled after the one described by Olton and Samuelson (1976, Experiment 2). The entire maze measured $183 \mathrm{~cm}$ across with the eight arms radiating at $45^{\circ}$ angles from an octagonal center platform ( $36 \mathrm{~cm}$ wide). All arms were $9.5 \mathrm{~cm}$ wide and $74 \mathrm{~cm}$ long. A recessed food well $(3.8 \mathrm{~cm}$ in diameter and $.6 \mathrm{~cm}$ deep) was located at the end of each arm, $67 \mathrm{~cm}$ from the center platform. A partition $(13 \mathrm{~cm}$ wide $\times 20 \mathrm{~cm}$ high) extended along one side of each arm at the point where the arm joined the center platform. A superstructure was mounted over the center platform and contained black Plexiglas guillotine doors $(9 \mathrm{~cm}$ wide $\times 10 \mathrm{~cm}$ high) and a removable transparent Plexiglas roof. Except for the black doors and black Plexiglas panels between door guides, the entire maze was painted white. The maze was mounted on legs such that the surface of the arms was $64 \mathrm{~cm}$ above the floor. The room in which the maze was housed was relatively rich in extramaze stimuli, containing, for example, water faucets and steam hoses on one wall and a shelf holding other pieces of apparatus on another wall. The room was normally illuminated by a single $40-\mathrm{W}$ incandescent bulb suspended $157 \mathrm{~cm}$ over the center of the maze. The experimenter stood in the doorway of the room and operated the guillotine doors via an overhead system of fishlines.

Procedure. By virtue of prior training, much as that described by Olton and Samuelson (1976), the rats were performing accur- 
ately in the eight-arm maze. Prior to each day's trial, each food well was baited with four $45-\mathrm{mg}$ pellets. The rat was then placed in the center of the maze and all doors were raised. A choice was recorded when the rat traversed the full length of an arm to the point of the food well. When the rat returned to the center platform after the fourth choice, the doors were lowered. After a delay containing treatments later described, the doors were raised and the rat was allowed to make additional choices until the rewards had been consumed from all eight arms. The entire trial rarely took more than $2-3 \mathrm{~min}$, and, as shall be seen, the rats rarely made a mistake (defined as returning to a previously chosen arm containing no food).

\section{Experiment 1a}

Variations in illumination during retention intervals are known to affect the performance of monkeys (D'Amato, 1973) and pigeons (Grant \& Roberts, 1976; Maki et al., 1977) in DMTS tasks. The question explored here was whether rats' memories established during the first few choices in the eight-arm maze would be similarly sensitive to variations in illumination during retention intervals. Zoladek and Roberts (1978) reported that rats' radial-arm maze performances suffer after blinding, implying that visual cues are important to successful performance. Such cues might then serve as mnemonic aids when the rat is exposed to them during retention intervals (as in Olton \& Samuelson, 1976). But Zoladek and Roberts reported that removal of sighted rats from the maze during retention intervals appeared to have little effect on postdelay choices. The present experiment, using sighted rats, manipulated the visual environment during retention intervals by manipulating illumination levels.

\section{Method}

Each rat performed in the eight-arm maze during each of three consecutive sessions. Each session consisted of a single trial as described above. Delays between the fourth and fifth choices were 2 min long. The overhead lighting during delays varied from day to day. The light was constantly on (as usual); it was turned off during the delay between the fourth and fifth choices; or it was manually switched off and on at about $1-\mathrm{sec} / \mathrm{change}$ during the delay. The three conditions were counterbalanced with respect to order of occurrence.

\section{Results}

No errors (defined as reentries into previously chosen arms) were made during the four choices prior to the delay. The mean accuracies during the first four choices following delays were $90 \%$ correct (light), 94\% (alternating light and dark), and $85 \%$ (all dark). An analysis of variance showed that the small effects of delay-interval illumination were not reliable $[\mathrm{F}(2,22)=1.26]$.

\section{Experiment 1b}

The failure of variations in illumination to interfere with rats' memories in the radial maze task can be explained in a variety of ways. The one examined here suggests that the rats maintained memories during the delay by a process analogous to "rehearsal" (cf. Maki et al., 1977), and that the variations in lighting were not sufficiently salient to capture attention and thereby disrupt rehearsal and promote forgetting. If true, one might expect variations along other, possibly more salient stimulus dimensions to be more disruptive. For example, Wallace, Steinert, Spear, Scobie, and Howard (Note 1) reported that rats are better at performing delayed conditional discriminations when the to-be-remembered cue is auditory rather than visual; the implication is that auditory events are more salient for rats. On the other hand, Zoladek and Roberts (1978) reported little effect of constant white noise on radial-arm maze performance. In this experiment, the auditory environment during the delay was manipulated by presenting white noise during the retention interval either constantly or pulsed on and off.

\section{Method}

This experiment began immediately after Experiment 1a. A speaker was placed on the floor beneath the center platform through which white noise ( $80 \mathrm{~dB}$ re $20 \mu \mathrm{N} / \mathrm{m}^{2}$ ) was occasionally presented. The noise was produced by a BRS/LVE audio-generator controlled by solid-state circuitry. The experiment spanned 6 days. Two features of the delay were manipulated. First, delays were either $15 \mathrm{sec}$ or $2 \mathrm{~min}$ long. Second, the room was silent during the delay (as usual), constant white noise was presented, or the noise was randomly switched on and off such that, in each second of the delay, the likelihood of noise was $p=.5$. The six conditions resulting from the orthogonal combination of delay length and auditory condition were counterbalanced over days with respect to order of occurrence.

\section{Results}

Again, no mistakes were made during the first four choices, and errors were rare during the four postdelay choices. For short and long delays, respectively, the mean percentages correct during Choices 5-8 were 94 and 96 (constant noise), 96 and 98 (alternating noise and silence), and 94 and 90 (no noise). An analysis of variance confirmed that the small differences were not reliable ones. Auditory conditions did not affect accuracy $[\mathrm{F}(2,22)=1.50]$; neither did accuracy vary across delays or as a function of both variables (both Fs $<1$ ).

\section{Experiment 1c}

This experiment might be regarded as an attempt to combine delay-interval stimulation from several dimensions. The specific rationale behind the manipulations stemmed from the failure of darkness during the delay to disrupt performance in the eight-arm maze during Experiment 1a. If the rats' performance in the eight-arm maze depends on extramaze cues, it might be reasoned that the rats use these cues to bridge intratrial delays. That is, leaving the rat confined in the maze during delays leaves the rat exposed to cues that may render rehearsal more effective. The results of Experiment la suggest otherwise; 
darkness should have interfered with the use of extramaze cues as mnemonic aids, but it did not do so. We further investigated the importance of exposure to extramaze cues during the delay in this experiment. Each rat was simply removed from the experimental room during the delay, thus both (a) depriving the rat of the extramaze cues, and (b) exposing it to a concatenation of visual, auditory, tactile, vestibular, and olfactory stimuli (cf. Zoladek \& Roberts, 1978).

\section{Method \\ Immediately after completion of Experiment $1 \mathrm{~b}$, the two sessions comprising this experiment occurred. In each session, the rats were exposed to a single trial in the eight-arm maze as described earlier, but with the following procedural changes. During the 1st day, after the fourth choice, the doors were lowered and the experimenter then removed the rat from the maze, carried it down a hallway and through another room, placed the rat in its empty home cage, carried the rat back to the maze, and then raised the doors and let the rat complete the day's trial. The total time of this operation was recorded for each rat $(M=58 \mathrm{sec}$, range: 54-64). During the 2 nd day, each rat was simply confined to the center of the maze (as in Experiments 1a and 1b), but for the same delay that it experienced on the preceding day.}

\section{Results}

No mistakes were made during the first four choices, and few mistakes occurred during the postdelay choices. After removal and confinement, the average choice accuracies were $94 \%$ and $90 \%$ correct, respectively $[\mathrm{F}(1,11)=1.00]$.

\section{Experiment 1d}

It could still be argued that none of the preceding manipulations provided stimuli during the delays that were sufficiently salient. Thus, goes the argument, the rats ignored those intradelay stimuli and devoted memorial processing to maintaining traces established during the four predelay choices. In the present experiment, we sought to provide, during delay intervals, what we suspected would be an extremely salient stimulus for the hungry rat: food.

\begin{abstract}
Method
The experiment commenced immediately after the preceding one. For this experiment only, the eight-arm maze was modified in two ways; a metal food cup $(4.5 \mathrm{~cm}$ in diameter $\times 2.5 \mathrm{~cm}$ deep) was embedded in a 6.4-cm square block of wood and affixed to the center of the central platform, and a small piece of rubber tubing was inserted through a hole drilled in the center of the Plexiglas roof. During the 1st day, each rat was allowed the customary four choices before the doors were lowered. Then the experimenter entered the room and dropped four 45-mg pellets into the food cup via the rubber tube. After a total delay of $60 \mathrm{sec}$, the doors were raised and the day's trial completed. On the 2 nd day, the rats were simply confined to the central platform (with an empty food cup) for a 60 -sec delay.
\end{abstract}

\section{Results}

No mistakes occurred during the first four choices. All animals ate all four pellets during the 60 -sec delay on Day 1, and accuracy during the four postdelay choices was uniformly high $(92 \%$ correct) on each day $(F<1)$. Consumption of food during the retention interval, then, did not disrupt the rat's memories of predelay choices.

\section{Experiment 1e}

One reason why the rats showed resistance to RI in earlier experiments might be that certain intramaze cues were present both during storage (Choices 1-4) and retrieval (Choices 5-8). Such an intramaze cue might be an odor trail laid down by each rat during the first four choices. Prior work with the eight-arm maze offers little in the way of support for such a hypothesis. Olton and Samuelson (1976) bathed their maze with aftershave lotion and found little effect on performance. Zoladek and Roberts (1978) rendered some of their rats acutely anosmic and found no effect on performance, even when those rats were removed from the maze during the retention interval. Still, it could be argued that our rats utilized olfactory cues when exposed to delay-interval events, particularly since Olton and Samuelson conducted their olfactory masking study without a delay and Zoladek and Roberts' study contained only two animals per group. The present experiment addressed this issue in the following ways. First, the rats were removed from the maze during the delay and placed in an "RI chamber" in which lights and noises were randomly scheduled. Second, we followed Olton and Samuelson (1976) in washing the maze with aftershave lotion prior to each run. In addition, the aftershave lotion was refreshed during the delay. Third, we attempted to establish the effectiveness of the aftershave as an olfactory masker in an independent food-searching task. The rats were trained to find pieces of chocolate Oreo cookies buried in piles of woodshavings; this kind of task has been shown to be a sensitive assessment of behavioral deficits in rats made acutely anosmic (Alberts \& Galef, 1971; Zoladek \& Roberts, 1978). In total, then, these manipulations (a) removed the rat from both intra- and extramaze cues during the delay, (b) exposed the rat to a variety of sensory stimulation during the delay, (c) degraded possible olfactory cues in the maze, and (d) assessed the potency of that degradation.

\section{Method}

The same subjects served immediately after the preceding experiment. In addition to the eight-arm maze, two other pieces of apparatus were used. The RI chamber was a clear Plexiglas box with a grid floor $(20 \mathrm{~cm}$ square $\times 23 \mathrm{~cm}$ high). The chamber was located in the dark hallway just outside of (but out of sight of) the room housing the eight-arm maze. A 6-W incandescent bulb was mounted in the Plexiglas roof and the speaker mentioned earlier was placed next to the box. When noise was presented (via the audiogenerator and controlling circuitry), the sound pressure level was $80 \mathrm{~dB}$ re $20 \mu \mathrm{N} / \mathrm{m}^{2}$. The light and noise were independently and randomly switched on and off every second when the control circuit was activated. The other apparatus, a modified open field, was a flat black box $(51 \mathrm{~cm}$ square $x$ 
$22 \mathrm{~cm}$ deep) covered with a clear Plexiglas lid and filled to a depth of about $3.8 \mathrm{~cm}$ with wood shavings. The box was located in yet another, brightly lit, room.

During the first 13 days of the experiment, the rats were trained daily in the eight-arm maze for one trial/day with a $60-\mathrm{sec}$ delay between Choices 4 and 5 . On the 14th day, the rats were removed from the maze after the fourth choice, placed in the RI chamber and exposed to $40 \mathrm{sec}$ of light and noise, and then returned to the maze and allowed to complete the trial; the delays averaged $78 \mathrm{sec}$ (range: $74-80$ ). On the next day, the rats were simply confined to the central platform of the maze between fourth and fifth choices for the same delays experienced during the preceding day. On Day 16, the rats were again confined for those delays; the maze was bathed with Mennen's aftershave lotion before each trial. On Day 17, the rats were removed and placed in the RI chamber after the fourth choice and exposed to $40 \mathrm{sec}$ of light and noise; the maze was bathed with the aftershave prior to each trial and rebathed during the delay. Delays averaged 92 sec (range: 84-108) on Day 17.

Concealed cookie testing occurred after each rat's daily maze trial. On Day 1, the rat was provided about one-quarter of a Nabisco chocolate Oreo cookie (about $2.8 \mathrm{~g}$ ) in the home cage to familiarize the rat with the food; all rats consumed the cookie. After the maze trial on Day 2, one-quarter cookie was placed on the surface of the bedding in the open-field box at locations chosen at random and varied across rats. The rat was placed in the apparatus and allowed to find, grasp, and begin to eat the cookie; the rat was then removed and allowed to consume the cookie in the home cage. The training during Days 3-14 was conducted in like manner, except that the piece of cookie was concealed at random locations about $2.5 \mathrm{~cm}$ beneath the surface of the shavings. During Days 15 and 17, Mennen's aftershave lotion was liberally sprinkled on the surface of the shavings before each rat's training and the piece of cookie was placed on the surface of the shavings. On Day 16, the cookies were concealed beneath the surface after the aftershave application. During these latter 3 days, each rat was allowed $5 \mathrm{~min}$ to find, grasp, and begin eating the cookie; if the cookie was not found within $5 \mathrm{~min}$, a failure was scored, and the rat and cookie were returned to the home cage.

\section{Results}

During the first 13 days (while the rats were being trained for the cookie test), no mistakes were made during the first four choices in the eight-arm maze and performances averaged $95 \%$ correct during the second four choices. During testing (Days 14-17), no errors were made on the first four choices and performances were highly accurate during the second four postdelay choices. When simply confined to the center of the maze after the fourth choice (Day 15), performance was perfect $(100 \%)$. After removal and exposure to the RI chamber (Day 14), $96 \%$ of the second four choices were correct. The corresponding values with added odor (Days 16 and 17) were $92 \%$ and $96 \%$. An analysis of variance confirmed that there was no overall effect of interference $(F<1)$, that the small effects of added odor $(98 \%$ vs. $94 \%)$ were unreliable $[F(1,11)=2.67]$, and that the degree of interference did not depend on presence or absence of odor $[F(1,11)=2.00]$.

Mean times to find, grasp, and begin eating the concealed cookie decreased from $66 \mathrm{sec}$ (range: 21-307) on Day 3 to $16 \mathrm{sec}$ (range: 4-39) on Day 14. When the cookie was placed on the surface of the odorized bedding on Day 15, the mean time significantly increased to $88 \mathrm{sec}$ (range: 16-243), as assessed by a Wilcoxon rank-sum test $[T=3, p<.01]$, but all animals found the cookie within the allotted time $(300 \mathrm{sec})$. When the cookie was buried in the odorized bedding on Day 16, the mean time further increased to $286 \mathrm{sec}$ [ $\mathrm{T}=0, \mathrm{p}<.01$ ], with 10 of the 12 rats failing to find the cookie within $300 \mathrm{sec}$ (thus receiving a score of $300 \mathrm{sec}$ by default). When the cookie was again placed on the odorized surface on Day 17 , no animal failed to find the cookie; latencies averaged $18 \mathrm{sec}$, a value less than that in the first surface test $(88 \mathrm{sec})[\mathrm{T}=0, \mathrm{p}<.01]$, but not significantly different from that during the final training day $(16 \mathrm{sec})$ $(\mathrm{T}=42)$.

\section{Discussion}

The results from the different parts of this experiment suggest that spatial memories formed by rats in the radial-arm maze may be resistant to several potential sources of RI. First, other work by Zoladek and Roberts (1978) suggests that visual cues are important to radial-arm maze performances. Yet, darkening the room during the retention interval or removing the rat from the maze did not disrupt performance. If visual cues are important, it appears that the rat need not be exposed to them during retention intervals in order to utilize them to guide postdelay choices. Second, auditory stimuli might be more salient for rats than visual stimuli (Wallace et al., Note 1), but fluctuations in the auditory delayinterval environment did not harm maze performance (see also Zoladek \& Roberts, 1978). Third, auditory and visual stimuli were shown to be just as ineffective amnesic agents in combination as they were singly. Fourth, food presented during the delay also failed to disrupt postdelay choices. It thus becomes more difficult to argue that RI was not obtained for reasons of inattention to delay-interval stimuli, because the hungry rats observed and consumed (and presumably attended to) the delay-interval food and yet showed no signs of RI. Taken together, these results suggest the possibility that the preservation of spatial memories in our experiments may not demand much, if any, of the rat's attention. Finally, bathing the maze with odor did not disrupt accuracy of choices prior to or following a delay. Nor did the odor potentiate the effects of interference during the delay. As assessed by the concealed cookie test, the same odor severely disrupted olfaction-guided food searching behavior. That the same odor did not disrupt eight-arm maze performance supports the conclusion that intramaze olfactory cues are relatively unimportant (Olton, 1978; Zoladek \& Roberts, 1978). The implication is that the resistance to RI shown by the animals in these experiments did not occur because 
of olfactory cues which persisted during and beyond the delay.

\section{EXPERIMENT 2}

Based on the data from Experiment 1, it might be concluded that rats' memories for "places passed" (Olton \& Samuelson, 1976) are resistant to RI from incidental stimuli. If, however, rats' memories for initial choices were coded along none of the manipulated stimulus dimensions but rather in terms of space, there would be no reason to expect that any of the preceding manipulations would have been effective in disrupting those memories. That is, it may be that only spatial stimuli (or reuse of a spatial memory system) will retroactively interfere with other spatial memories. Consequently, the present experiment explored the possibility that another spatial task might prove to be a more effective source of RI. The rats were trained to perform in a four-arm maze and that task was then interpolated between the fourth and fifth choices in the eight-arm maze.

\section{Method}

The rats used in the earlier experiment served here. They were allowed unlimited access to food in home cages for 23 days after Experiment 1 and then reduced to $85 \%$ of their new free-feeding weights. Another four-arm maze was constructed, which measured $137 \mathrm{~cm}$ across with all arms $9.5 \mathrm{~cm}$ wide and $53 \mathrm{~cm}$ long, radiating at $90^{\circ}$ angles from a 30 -cm square central platform. A recessed food well was located at the end of each arm. The superstructure mounted over the central platform contained clear Plexiglas guillotine doors $(9.5 \mathrm{~cm}$ wide $\times 18 \mathrm{~cm}$ high) and a clear Plexiglas roof. The door guides were $7.6 \mathrm{~cm}$ wide and the spaces between guides (the corners of the platform) were left open. Except for the doors and roof, the entire maze was coated with a natural wood (pecan) stain. The maze was mounted on legs such that the surface of the maze was $53 \mathrm{~cm}$ above the floor. This apparatus was illuminated by a $40-\mathrm{W}$ incandescent bulb suspended $137 \mathrm{~cm}$ above the central platform. The maze was located in the end of a hallway just outside of the room housing (but out of sight of the eight-arm maze. This area, too, was rich in extramaze cues, including storage cabinets, a rack of empty cages, and the generally motionless experimenter, who operated the guillotine doors via another overhead system of lines.

All 12 rats were first trained in the four-arm maze. The training procedures were similar to those used with an eight-arm maze, except that the rewards were two 45-mg pellets placed in the food well on each arm. Each rat was placed in the center compartment, the doors raised, and the rat allowed to make choices until all rewards were consumed or until $10 \mathrm{~min}$ had passed. Training continued for 2 days at 1 trial/day. During the next phase, each animal performed one eight-arm trial (with a 60 -sec delay between Choices $\mathbf{4}$ and 5) and one four-arm trial (with no delay). The runs in the different mazes were separated by about $1 \mathrm{~min}$, and the order of trials (four-arm first vs. eight-arm first) was alternated across the next 5 days of training. The times to make four choices averaged $30 \mathrm{sec}$ (range: 18-47) on the last training day in the fourarm maze. For unknown reasons, one rat began failing to complete the eight-arm trial within 10 min on Days 4 and 5 and was dropped from the experiment.

The remaining 11 animals were tested on a single day (Test $A)$. All rats began by making four choices in the eight-arm maze. They were then removed and allowed four choices in the four-arm maze, after which they were returned to the eight-arm maze and allowed to complete the trial. The total delays between fourth and fifth choices in the eight-arm maze averaged $87 \mathrm{sec}$ (range: 74-111).

During the next 8 days, training continued as described above (one trial on each maze in succession) but with a 30 -sec delay between Choices 2 and 3 in the four-arm (during which the rat was confined to the central platform). Then two replications of Test B were conducted. During the 1st (interference) day of each replication, the procedures of Test $A$ were followed (except for the 30 -sec delay in the four-arm maze). During the 2nd (control) day of each replication, the metal food cup described earlier (Experiment 1d) was fastened to the center of the four-arm maze and filled with eight $45-\mathrm{mg}$ pellets. The rats were then simply confined to the central platform of the four-arm maze for the same delays between Choices 4 and 5 of the eight-arm maze as experienced during the preceding day. For the two replications, the delays averaged $106 \mathrm{sec}$ (range: 99-116) and $105 \mathrm{sec}$ (range: 92-124). During the first replication, one rat became ill and was dropped from the experiment; the Test $B$ data are therefore based on 10 rats.

\section{Results}

During Test A, no errors were committed in the four-arm maze or during the first four choices in the eight-arm maze. During the second four choices, performances averaged $95 \%$ correct, a value not significantly different from that during the immediately preceding training day $(98 \%)(\mathrm{F}=1)$.

During Test B, no errors were committed during the first four choices in the eight-arm maze or during the first two choices in the four-arm maze. Performances during the second four choices in the eightarm maze averaged $94 \%$ and $96 \%$ correct during the interference and control days, respectively $(F<1)$. Performances of the second two choices in the fourarm maze during interference days averaged $75 \%$ correct, a value not significantly different from the $80 \%$ correct choices during the last training day prior to Test $B(F<1)$. During control days, most pellets were consumed in the four-arm maze $(\mathbf{M}=$ 7.75 , range: $6-8$ ).

\section{Discussion}

It could be charged, in connection with Experiment 1 , that RI is most likely to occur when the interfering stimuli are from the same stimulus dimension as the to-be-remembered stimuli. For example, Worsham and D'Amato (1973) found that delayinterval illumination (but not delay-interval auditory events) interfered with retention of visual samples in DMTS. So, if rats coded choices in terms of space, nonspatial manipulations might prove ineffective. The data from the present experiment, however, show that another spatial task interpolated during the delay does not interfere with eight-arm maze performance. The data also provide no evidence for "memory load" effects. That is, retention of the first four choices of the eight-arm task did not impair performance of the four-arm task. Neither did increased memory requirements in the four-arm task 
(Task A with no delay vs. Test B with a $30-\mathrm{sec}$ delay) produce interference with retention of the first choices of the eight-arm task.

Regardless of how the question of retroactive interference with rats' spatial memories is resolved, the present findings provide grounds for increasing our estimates of rats' cognitive capabilities. Not only do rats perform very well on one spatial memory task, but they seem to do as well on two spatial memory tasks. Olton and Samuelson (1976, Experiment 6) administered multiple eight-arm trials within single sessions and found that the rats were nearly as proficient after eight trials as they were on the first trial, suggesting that the rats somehow managed to segregate the memories that were successively established in different trials, minimizing proactive interference. Similarly, our rats were able to segregate the spatial memories associated with the different mazes, minimizing retroactive interference. One possible interpretation of our results is that the rat is capable of processing spatial memories established in different contexts in parallel and without loss. The limits on such processing remain to be established.

\section{REFERENCE NOTE}

1. Wallace, J. E., Steinert, P., Spear, N. E., Scobie, S.R., \& Howard, S. Modality effects in the short-term retention of an operant conditional discrimination in rats. Paper presented at the meeting of the Midwestern Psychological Association, Chicago, May 1978.

\section{REFERENCES}

Alberts, J. R., \& Galef, B, G, JR. Acute anosmia in the rat: A behavioral test of a peripherally-induced olfactory deficit. Physiology \& Behavior, 1971, 6, 619-621.

D'Amato, M. R. Delayed matching and short-term memory in monkeys. In G. H. Bower (Ed.), The psychology of learning and motivation: Advances in research and theory (Vol. 7). New York: Academic Press, 1973.

Grant, D. S., \& Roberts, W. A. Sources of retroactive inhibition in pigeon short-term memory. Joumal of Experimental Psychology: Animal Behavior Processes, 1976, 2, 1-16.

Herman, L. M. Interference and auditory short-term memory in the bottle-nosed dolphin. Animal Learning \& Behavior, 1975, 3, 43.48.

Maki, W. S., Moe, J. C., \& Bierley, C. M. Short-term memory for stimuli, responses, and reinforcers. Journal of Experimental Psychology: Animal Behavior Processes, 1977, 3, 156-177.

Olton, D. S. Spatial memory. Scientific American, 1977, 236, 82-98.

Olton, D. S. Characteristics of spatial memory. In S. H. Hulse, H. Fowler, \& W. K. Honig (Eds.), Cognitive processes in animal behavior. Hillsdale, N.J: Erlbaum, 1978.

Olton, D. S., \& Samuelson, R. J. Remembrance of places passed: Spatial memory in rats. Journal of Experimental Psychology: Animal Behavior Processes, 1976, 2, 97.116.

Worsham, R. W., \& D'Amato, M. R. Ambient light, white noise, and monkey vocalization as sources of interference in visual short-term memory of monkeys. Journal of Experimental Psychology, 1973, 99, 99-105.

Zoladek, L., \& RoBerts, W. A. The sensory basis of spatial memory in the rat. Animal Learning \& Behavior, 1978, 6, 77-81.

(Received for publication June 13, 1978; revision accepted August 12, 1978.) 